\title{
Análisis del Clima Laboral en el Instituto Superior Tecnológico Juan Bautista Aguirre en Tiempos de Pandemia
}

\author{
Darwin Vicente Apolo Robles \\ Marcel Oswaldo Méndez-Mantuano \\ Keyla Ximena Bodero Jiménez. \\ Soraya Francisca Alvarado Fiallo \\ Ángel Raúl Huayamave Rosado
}

Instituto Superior Tecnológico Juan Bautista Aguirre, Ecuador

Doi:10.19044/esj.2022.v18n1p54

Submitted: 21 October 2021

Accepted: 07 January 2022

Published: 31 January 2022
Copyright 2022 Author(s)

Under Creative Commons BY-NC-ND

4.0 OPEN ACCESS

Cite As:

Apolo Robles D.V., Méndez-Mantuano M.O., Bodero Jiménez K.X., Alvarado Fiallo S.F. \& Huayamave Rosado A.R. (2022). Análisis del Clima Laboral en el Instituto Superior Tecnológico Juan Bautista Aguirre en Tiempos de Pandemia. European Scientific Journal, ESJ, 18 (1), 54. https://doi.org/10.19044/esj.2022.v18n1p54

\section{Resumen}

La pandemia sigue afectando al componente social de las personas, debido a las restricciones, los distanciamientos y los confinamientos, provocando cambios drásticos en la forma de convivir con el entorno y en las interrelaciones laborales. Con estas perspectivas surge la necesidad de conocer y evaluar los entornos laborales durante la pandemia, con el objetivo de conocer las particularidades y conflictos que se desarrollan en esta nueva relación laboral. La educación se ha adaptado a la modalidad de teletrabajo para los docentes y de teleeducación para los estudiantes, donde ambos grupos humanos han tenido que ajustar los entornos de su residencia para desarrollar las diferentes actividades. La presente investigación tiene como propósito analizar el clima laboral en el Instituto Superior Tecnológico Juan Bautista Aguirre de la ciudad de Daule (Ecuador) durante la pandemia de covid-19; para ello se realizó una encuesta a 61 docentes voluntarios, en la cual se abordaron las dimensiones de: sentido de pertenencia, relaciones entre compañeros, satisfacción, eficacia y eficiencia, liderazgo, reconocimiento y oportunidades de desarrollo e innovación. Adicionalmente se realizó una entrevista a la máxima autoridad de la institución para conocer las perspectivas gerenciales relacionadas al clima laboral. De manera general se determinó el 
promedio porcentual de las dimensiones, donde el $73 \%$ de la valoración fue positiva, mientras que el $27 \%$ fue negativa.

Palabras claves: Clima laboral, dimensiones, pandemia, valoración

\title{
Analysis of the Working Climate at the Juan Bautista Aguirre Institute of Technology in Times of Pandemic
}

\author{
Darwin Vicente Apolo Robles \\ Marcel Oswaldo Méndez-Mantuano \\ Keyla Ximena Bodero Jiménez \\ Soraya Francisca Alvarado Fiallo \\ Ángel Raúl Huayamave Rosado
}

Instituto Superior Tecnológico Juan Bautista Aguirre, Ecuador

\begin{abstract}
The pandemic continues to affect the social component of people, due to restrictions, distancing, and confinement, causing drastic changes in the way of living with the environment and in work interrelationships. With these perspectives arises the need to know and evaluate the work environments during the pandemic, with the aim of knowing the particularities and conflicts that develop in this new employment relationship. Education has been adapted to the modality of telework for teachers and tele-education for students, where both human groups have had to adjust the environments of their residence to develop the different activities. The purpose of this research is to analyze the work environment at the Juan Bautista Aguirre Higher Technological Institute in the city of Daule (Ecuador) during the covid-19 pandemic; For this, a survey was carried out on 61 volunteer teachers, in which the dimensions of: sense of relevance, peer relationships, satisfaction, effectiveness and efficiency, leadership, recognition and opportunities for development and innovation were addressed. Additionally, an interview was conducted with the highest authority of the institution to learn the managerial perspectives related to the work environment. In general, the average percentage of the dimensions was determined, where $73 \%$ of the assessment was positive, while $27 \%$ was negative.
\end{abstract}

Keywords: Work environment, dimensions, pandemic, assessment

\section{Introduction}

Las metodologías de trabajo siguen evolucionando de la mano de las nuevas tecnologías, las cuales permiten incrementar la productividad en todos 
los ámbitos dentro de la administración de una empresa o institución. Sin embargo, aún persiste un tema sin resolver, el mismo que está ampliamente abordado y es de vital importancia para el correcto desarrollo organizacional, el denominado clima o ambiente laboral. Este aspecto se sigue desarrollando de parte de muchos investigadores, sociólogos, catedráticos, gerentes y empleados de las diferentes organizaciones públicas y privadas, ya que tener un buen "ecosistema laboral" mejora todos los aspectos y procesos dentro de las mismas (Revista Líderes, 2018).

Los trabajadores poseen ideas preconcebidas sobre sí mismos y de su ambiente, las cuales reaccionan con el entorno donde trabajan, tales como su jefe inmediato, la rigidez de la estructura organizacional y las opiniones de los grupos de trabajos. Todo esto conlleva a que el desempeño de los empleados dependa del análisis objetivo de las circunstancias y las impresiones subjetivas del ambiente donde labora. Aquellas empresas o instituciones que no sean capaces de reclutar o mantener una fuerza laboral eficaz, serán aquellas que poseen menores niveles de crecimiento económico o de desarrollo institucional (Dessler \& Varela, 2011).

De manera irrefutable las personas intercambian información con el medio y crean un equilibrio dinámico con él, diseñando actitudes de correspondencia con el mismo, por ello, los espacios laborales son también entornos psicológicos, en donde convergen las percepciones personales y colectivas, y de esta manera se establece la conducta organizacional de todos los trabajadores (Acosta \& Venegas, 2010).

En 1968 Litwin y Stringe fueron los primeros en conceptualizar el clima laboral desde la visión perceptual de cada uno de los miembros de una empresa, quienes establecieron que dicho ambiente es la suma de todos los elementos del entorno laboral, los cuales consiguen ser medidos de forma directa e indirecta y que pueden verse alterados por los comportamientos de los otros miembros o por las motivaciones internas. Estos autores propusieron la existencia de nueve dimensiones que explicarían el clima laboral, las cuales son: estructura, responsabilidad, recompensa, riesgo, calor, apoyo, estándares de desempeño, conflicto e identidad (Acosta \& Venegas, 2010).

Uno de los grandes retos del siglo 21 es mejorar las relaciones laborales, no tan solo en sus condiciones estructurales o en lo referente a la escala salarial, sino en el ambiente que perciben las personas al realizar sus actividades habituales. Un buen ambiente genera sentimientos positivos de autorrealización mejorando la autoestima, ya que existe satisfacción de los colaboradores al saber que aportan de forma significativa a su organización, repercutiendo directamente en su calidad de vida (Bobadilla, 2017).

Se define como clima laboral las diferentes percepciones que poseen los empleados en relación con la satisfacción de las necesidades sociales y del buen sentimiento de la labor que realiza, por lo tanto, el análisis crítico del 
ambiente laboral permite interpretar las situaciones para posteriormente reorientar las acciones, con el propósito de lograr la satisfacción de los involucrados (Moreno \& Pérez, 2018).

Un buen ambiente de trabajo crea en los colaboradores un sentido de identidad y pertenencia, generando un compromiso más amplio con la organización, donde las reglas estrictas son un factor secundario para la realización de las actividades. Esto confluye en la creación de un capital social humano con elevadas características laborales, quienes buscan constantemente el bienestar para la organización y en las relaciones interpersonales. Lo anteriormente indicado no niega los posibles conflictos, sino que las posiciones individuales suelen mimetizarse a través de los intereses colectivos (Daft, 2011).

El clima laboral no es un factor homogéneo ni estático dentro de una organización, donde existen "microclimas" en cada uno de los diferentes departamentos, por lo tanto, la suma de todos los microclimas corresponde al clima organizacional. Dentro del mismo sobresalen aspectos como la multidimensionalidad y la sectorialidad, el primero hace referencia a la diversidad de aspectos que componen el clima, mientras que el último hace relación a la variabilidad existente dentro de una misma unidad administrativa (García, 2006).

Dentro de una organización los individuos se comportan de acuerdo con los estímulos internos y externos percibidos, los cuales inciden en la productividad, en el sentido de pertenencia, en la calidad del servicio, en la efectividad, en su eficacia y en el impacto social que se genera. De manera general, el clima laboral afecta de manera positiva o negativa en el desempeño de toda la organización, de tal manera que se busca reducir los posibles conflictos entre la parte administrativa y la operativa (Salazar et al., 2009).

El clima laboral no es una moda reciente, sino que es una preocupación recurrente de las instituciones, el mismo se constituye como un elemento de la calidad laboral de sus trabajadores, pero que ha tomado nuevas connotaciones a partir de la pandemia que se inició en el 2020, debido a que muchos trabajadores cambiaron su forma laboral de la presencialidad a la virtualidad (teletrabajo), por lo tanto, existen nuevas particularidades que se deben establecer para conocer el "clima laboral" desde la virtualidad de las actividades. Por ello, la investigación analiza la percepción del clima laboral de los docentes del Instituto Superior Tecnológico Juan Bautista Aguirre de la ciudad de Daule, a partir de la aplicación de una entrevista a la máxima autoridad institucional y en el desarrollo de una encuesta basada en varios elementos del cuestionario de clima organizacional de Litwin y Stringer (1968) y en las dimensiones de Bizneo (2021), Litwin y Stringer (1968) y de Patterson et al. (2005). 


\section{Metodología}

El enfoque de la investigación es mixto, debido al análisis de los porcentajes proporcionados por la aplicación de encuestas a 61 docentes voluntarios del Instituto Superior Tecnológico Juan Bautista Aguirre, y a la realización de la entrevista a la doctora Ida Campi Mayorga, quien se desempeña como máxima autoridad de la institución objeto de estudio. Esta investigación tomó elementos de los estudios realizados por Miguel (2020).

El diseño es no experimental con elementos longitudinales de evolución de grupo (docentes). La información para el análisis del clima laboral proviene de fuentes primarias (entrevista a la autoridad institucional objeto de estudio y a las encuestas proviene de los docentes), las cuales se realizaron a través de Google Forms (encuestas) y de la plataforma Zoom (entrevista).

Las preguntas aplicadas son las siguientes:

1 ¿Se siente bien en su trabajo?

- $\mathrm{Si}$

- A veces

- No

2 ¿Siente plena satisfacción laboral bajo la modalidad virtual?

- $\mathrm{Si}$

- A veces

- No

3 ¿Qué modalidad laboral prefiere?

- Presencial

- Virtual

4 ¿Cuál es su nivel de satisfacción de manera regular mientras realiza las labores docentes?

- Muy satisfecho

- Satisfecho

- Neutro

- Insatisfecho

- Muy insatisfecho

5 ¿Cuál es su nivel de satisfacción de manera regular mientras realiza las labores administrativas?

- Muy satisfecho

- Satisfecho

- Neutro

- Insatisfecho

- Muy insatisfecho

6 ¿Tiene claro lo que se espera de su trabajo?

- $\mathrm{Si}$ 
- A veces

- No

7 ¿Considera que su esfuerzo ha tenido el justo reconocimiento?

- $\mathrm{Si}$

- A veces

- No

8 ¿Los objetivos de la institución reflejan sus intereses?

- $\mathrm{Si}$

- No

9 ¿Considera que la carga laboral docente es la idónea para usted?

- $\mathrm{Si}$

- No

10 ¿Las directrices para realizar un determinado trabajo asignado son claras?

- Si

- A veces

- No

11 ¿Los plazos dados para realizar y entregar un trabajo están proporcionalmente establecidos?

- $\mathrm{Si}$

- A veces

- No

12 ¿Existe flexibilidad para la entrega de trabajo atrasado que le fue asignado?

- $\mathrm{Si}$

- A veces

- No

13 ¿De manera regular es interrumpido en sus horas fuera de la jornada laboral?

- $\mathrm{Si}$

- A veces

- No

14 ¿Considera que el clima laboral de la institución se ha deteriorado durante la pandemia?

- $\mathrm{Si}$

- No

15 ¿Existe un ambiente de cordialidad que permita la retroalimentación constante entre las autoridades y compañeros?

- $\mathrm{Si}$

- A veces

- No 
16 ¿Cree que tiene oportunidades para crecer profesionalmente en la institución?

- $\mathrm{Si}$

- No

17 ¿Su jefe directo es justo en sus decisiones?

- $\mathrm{Si}$

- A veces

- No

18 ¿Ha tenido oportunidades de innovar en su trabajo?

- $\mathrm{Si}$

- A veces

- No

19 ¿Considera que existen sesgos de parte de las autoridades para favorecer a ciertas personas?

- $\mathrm{Si}$

- No

20 ¿Existe una buena relación con sus compañeros de trabajo?

- $\mathrm{Si}$

- A veces

- No

21. De manera general el clima laboral de la institución es

- Muy satisfactorio

- Satisfactorio

- Regular

- Malo

- Muy Malo

El alcance es descriptivo, donde no se profundiza en las causas efectos de los resultados en cada una de las dimensiones, sino que se toma una postura imparcial sobre el clima laboral óptimo (presencial o virtual).

Para analizar y procesar la información se elaboraron tablas y gráficos estadísticos que representan los porcentajes de cada dimensión. La encuesta constaba de 21 preguntas y se utilizó la escala de Likert para determinar el grado de preferencia en cada una de las preguntas. Para determinar los niveles porcentuales positivos y negativos de cada dimensión, se estableció la siguiente valoración: 
Tabla I. Valoración positiva o negativa de las diferentes escalas usadas en la encuesta

\begin{tabular}{|c|c|c|c|}
\hline Escala & $\begin{array}{c}\text { Emoticón usado } \\
\text { en encuesta }\end{array}$ & Valoración & Observaciones \\
\hline $\mathrm{Si}$ & & Positiva & \multirow{8}{*}{$\begin{array}{l}\text { La pregunta } 3 \text { fue } \\
\text { excluida } \\
\text { determinar } \\
\text { valoración de la } \\
\text { dimensión } \\
\text { satisfacción. } \\
\text { En las preguntas 13, } \\
14 \text { y 19, se consideró } \\
\text { como positivo a los } \\
\text { porcentajes de las } \\
\text { respuestas con "no" }\end{array}$} \\
\hline A veces & & $\begin{array}{c}\text { División entre } \\
\text { positiva y negativa }\end{array}$ & \\
\hline No & & Negativa & \\
\hline Muy satisfecho & & Positiva & \\
\hline Satisfecho & & Positiva & \\
\hline Neutro & & $\begin{array}{c}\text { División entre } \\
\text { positiva y negativa }\end{array}$ & \\
\hline Insatisfecho & & Negativa & \\
\hline Muy insatisfecho & & Negativa & \\
\hline
\end{tabular}

A través de los promedios de los porcentajes de cada una de las dimensiones se estableció la valoración positiva y negativa, proyectándose dicho valor como el indicador numérico para el clima laboral.

Tal como fue mencionado anteriormente, el análisis del clima laboral en el Instituto Superior Tecnológico Juan Bautista Aguirre se basó en las dimensiones de Bizneo (2021), Litwin y Stringer (1968) y de Patterson et al. (2005), dado que existen paralelismos entre sus elementos y las particularidades del desarrollo laboral de los docentes en la institución objeto de estudio durante el confinamiento por la pandemia de covid-19. El establecimiento de las dimensiones permitió la estructuración de las encuestas aplicadas. Siendo las siguientes dimensiones analizadas:

\section{Dimensiones del Clima Laboral}

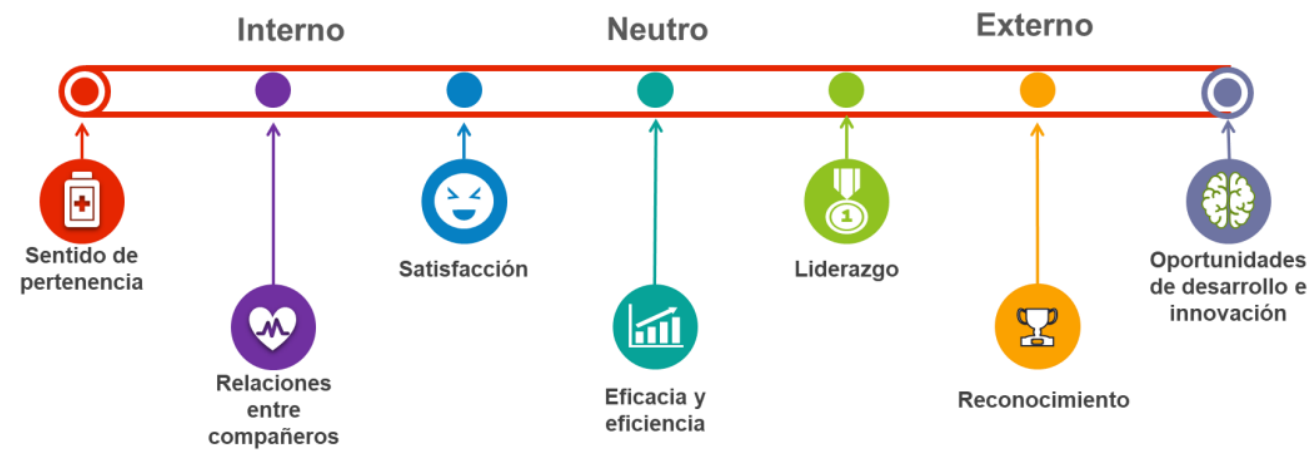

Figura 1. Dimensiones establecidas para medir el clima laboral en el ISTJBA. Fuente: los autores 
Las características que considera de cada dimensión son las siguientes:

1. Sentido de pertenencia: compromiso del colaborador con la institución, donde los objetivos de esta son considerados como propios.

2. Relaciones entre compañeros: percepción de bienestar y cordialidad en las relaciones sociales entre los miembros de la institución.

3. Satisfacción: sentimientos de bienestar al realizar las actividades inherentes al trabajo.

4. Eficacia y eficiencia: percepción de la capacidad para realizar una actividad de forma clara, dentro de plazos establecidos y extendidos.

5. Liderazgo: percepción de la capacidad en la toma de decisiones de parte de las autoridades, de forma ordenada y justa.

6. Reconocimiento: conocimiento de la valía que le otorga la institución a las personas.

7. Oportunidades de desarrollo e innovación: percepción del crecimiento personal y permeabilidad institucional para establecer diferentes opciones laborales.

Adicionalmente las dimensiones fueron divididas en internas, neutras y externas, definiéndose los criterios para esta agrupación de la siguiente manera:

1. Interna: son aquellas percepciones que de manera general se establecen dentro de la psiquis de las personas, las cuales pueden ser modificadas por las dimensiones externas.

2. Neutra: aquí se consideró la dimensión de eficacia y eficiencia, debido a que las opiniones de esta pueden ser moldeadas de forma rápida por las dimensiones internas y externas.

3. Externa: las dimensiones externas son los estímulos que captan las personas en las diferentes interrelaciones que desarrollan, básicamente es la percepción de las condiciones que diseñan terceros para desarrollar su trabajo.

Todas las dimensiones son dinámicas, por lo tanto, las percepciones dentro de un lapso de tiempo pueden ser alteradas de manera significativa en diferentes fechas o circunstancias.

En base a la anterior clasificación se separaron las preguntas de la encuesta: 
Tabla II. Clasificación de las preguntas por cada dimensión

\begin{tabular}{|c|c|c|}
\hline \multirow{2}{*}{ Interna } & Dimensiones & Preguntas \\
\cline { 2 - 3 } & Sentido de pertenencia & 6,8 \\
\cline { 2 - 3 } & Relaciones entre compañeros & 15,20 \\
\hline \multirow{2}{*}{ Neutra } & Satisfacción & $1,2,3,4,5,14$ \\
\hline \multirow{3}{*}{ Externa } & Eficacia y eficiencia & $9,10,11,12$ \\
\cline { 2 - 3 } & Liderazgo & $13,17,19$ \\
\cline { 2 - 3 } & Reconocimiento & 7 \\
\hline
\end{tabular}

\section{Resultados}

A continuación, se detalla el análisis por cada una de las dimensiones.

\section{Dimensiones internas}

El sentido de pertenencia promueve la proyección de la filosofía institucional en los colaboradores hacia el exterior, de tal manera que se identifican con la misma y logran un mejor desempeño de las funciones (Jurado, 2014). Los resultados estratificados por la dimensión de sentido de pertenencia indican que el 67,2\% de los docentes tiene definido de manera específica lo que se espera de su labor docente, por su parte el 80,3\% de los encuestados se ven identificados con los objetivos institucionales (tabla III).

Este sentido de pertenencia proviene de varios elementos internos, no exclusivamente de los mencionados en este apartado, sin embargo, el nivel de este varía según la sensación de seguridad que experimentan las personas al realizar sus actividades, esto conduce a que se busquen conductas que permitan ocupar o mantener un determinado lugar dentro de la institución (Chiavenato, 2009).

Tabla III. Resultados de la dimensión interna sentido de pertenencia

\begin{tabular}{|c|c|c|}
\hline \multicolumn{3}{|c|}{ ¿Tiene claro lo que se espera de su trabajo? } \\
\hline Sí & A veces & No \\
\hline $67,2 \%$ & $29,5 \%$ & $3,3 \%$ \\
\hline \multicolumn{2}{|c|}{ ¿Los objetivos de la institución reflejan sus intereses? } \\
\hline Sí & No \\
\hline $80,3 \%$ & $19,7 \%$ \\
\hline
\end{tabular}

Tal como fue mencionado anteriormente, para la valoración de los niveles positivos y negativos de cada una de las preguntas, se consideró positivo a los porcentajes con las respuestas afirmativas y negativas a los porcentajes opuestos (con las excepciones mencionadas en la metodología), mientras que los valores neutros (a veces) fueron divididos en partes iguales y asignados a los niveles positivos y negativos. La valoración de la dimensión fue establecida mediante el promedio de los valores positivos y negativos de cada pregunta que componen dicha dimensión. 


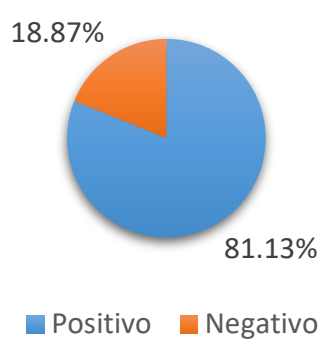

Figura 2. Valoración de la dimensión sentido de pertenencia

Esto evidencia que existe un alto sentido de pertenencia entre los miembros de la comunidad educativa de la institución objeto de estudio. Esta dimensión crea el compromiso entre los colaboradores y la institución, en eso radica su importancia.

La dimensión de relación entre compañeros es una de las más analizadas por los diferentes investigadores, ya que estas relaciones permiten crear vínculos sociales que fomentan la colaboración grupal para un determinado objetivo. Los altos niveles de compañerismo están directamente relacionados con los niveles de armonía interna, donde prevalece la comunicación, la confianza, la retroalimentación, etc.

Surge la pregunta ies posible crear o mantener relaciones sanas entre compañeros laborales en la virtualización de las relaciones sociales? Para ello, hay que interiorizar en los elementos que permiten mantener o crear este tipo de relaciones sociales. En este factor tampoco existe un consenso claro para identificar de manera precisa cuáles podrían ser los elementos, sin embargo, para el presente análisis se consideró la retroalimentación y las buenas relaciones de forma general (respeto, comprensión, amabilidad, y comunicación).

El 50,8\% de los docentes consideran que existe cordialidad en la institución, la misma que permite la retroalimentación entre las autoridades y sus pares. El $41 \%$ estimó que únicamente a veces existe este ambiente (tabla IV). Esto supondría que la comunicación virtual no permite crear una atmósfera de cordialidad para dicha retroalimentación. Posiblemente se debe a que la interrelación por los medios tecnológicos es puntual y no se llega a profundizar en elementos que permiten conocer la perspectiva de los pares, en otras palabras, existe comunicación unidireccional que no permite la retroalimentación, donde las personas se transforman en oidores de las disposiciones de la gerencia o de las autoridades.

A nivel horizontal, la relación de compañerismo es percibida de manera favorable en $83,6 \%$ de los encuestados, lo que indicaría que las relaciones de compañerismo y de amistad se han mantenido (incluso aumentado) durante la pandemia. Esto se debe presumiblemente a factores 
como la conectividad a través de plataformas sociales que son significativamente superiores, las cuales promueven relaciones informales que son características de la amistad, o debido a que los niveles de conflictividad de manera virtual son inferiores a los niveles de conflictividad de forma presencial (Condella, 2012).

Tabla IV. Resultados de la dimensión interna relación entre compañeros

\begin{tabular}{|c|c|c|}
\hline \multicolumn{3}{|c|}{ ¿Existe un ambiente de cordialidad que permita la retroalimentación constante entre las } \\
autoridades y compañeros?
\end{tabular}

La valoración positiva y negativa de la dimensión relación entre compañeros es:

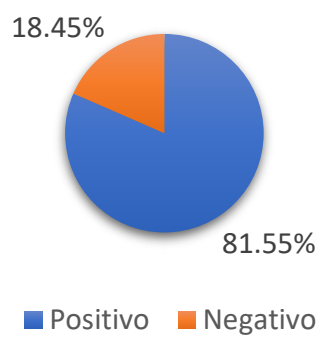

Figura 3. Valoración de la dimensión relación entre compañeros

Esta dimensión también es alta, lo cual demuestra que existe una elevada relación entre compañeros de la institución, y que es favorable la interacción durante la pandemia a través de las redes sociales informales.

En la dimensión de satisfacción se estimaron numéricamente varios aspectos, por ejemplo, la sensación de bienestar (al momento de efectuar la encuesta) equivalió al $80,3 \%$, la satisfacción en la realización de las actividades a través de la modalidad laboral virtual fue del 50,8\%, la preferencia en relación al tipo de modalidad laboral fue por la virtual en un $55,7 \%$, la satisfacción mientras se ejecutan actividades docentes es del 50,8\%, mientras que este porcentaje cae al $34,4 \%$ al realizarse actividades administrativas, finalmente la mayoría de las personas $(54,1 \%)$ consideran que no se ha deteriorado el ambiente laboral durante la pandemia (tabla V).

El porcentaje de personas que prefieren la modalidad laboral virtual es del 55,7\%. Esta misma pregunta fue abordada meses antes por Méndez et al. (2020) en la misma institución, teniendo como preferencia la modalidad presencial con un $64,6 \%$; lo que indicaría que a medida que transcurre el tiempo, un mayor número de docentes prefiere realizar sus actividades docentes y administrativas desde su hogar. 
También resalta que la satisfacción en las actividades de docencia es superior a la satisfacción en las actividades administrativas. Estas últimas corresponden a realización de syllabus, planes analíticos, informes de seguimiento, informes finales, informes como directores o gestores departamentales, informes como coordinadores o gestores de la coordinación, entre otros. Este bajo nivel de satisfacción podría corresponder a que las actividades administrativas son ejecutadas fuera de las jornadas laborales (presumiblemente), o debido a la rutina que representa poder realizarlas.

Tabla V. Resultados de la dimensión interna satisfacción

\begin{tabular}{|c|c|c|c|c|}
\hline \multicolumn{5}{|c|}{ ¿Se siente bien en su trabajo? } \\
\hline \multicolumn{2}{|l|}{ Sí } & A veces & \multicolumn{2}{|r|}{ No } \\
\hline \multicolumn{2}{|c|}{$80,3 \%$} & $19,7 \%$ & & 0 \\
\hline \multicolumn{5}{|c|}{ ¿Siente plena satisfacción laboral bajo la modalidad virtual? } \\
\hline \multirow{2}{*}{\multicolumn{2}{|c|}{$\frac{\text { Sí }}{50,8 \%}$}} & \multicolumn{2}{|l|}{ A veces } & No \\
\hline & & $42,6 \%$ & & $6,6 \%$ \\
\hline \multicolumn{5}{|c|}{ ¿Qué modalidad laboral prefiere? } \\
\hline \multicolumn{3}{|c|}{\begin{tabular}{l|l} 
Presencial & \\
\end{tabular}} & \multicolumn{2}{|c|}{ Virtual } \\
\hline \multicolumn{3}{|c|}{$44,3 \%$} & \multicolumn{2}{|c|}{$55,7 \%$} \\
\hline \multicolumn{5}{|c|}{$\begin{array}{l}\text { ¿Cuál es su nivel de satisfacción de manera regular mientras realiza las laborales } \\
\text { docentes? }\end{array}$} \\
\hline Muy satisfecho & Satisfecho & Neutro & Insatisfecho & $\begin{array}{c}\text { Muy } \\
\text { insatisfecho }\end{array}$ \\
\hline $50,8 \%$ & $37,7 \%$ & $11,5 \%$ & 0 & 0 \\
\hline \multicolumn{5}{|c|}{$\begin{array}{l}\text { ¿Cuál es su nivel de satisfacción de manera regular mientras realiza las laborales } \\
\text { administrativas? }\end{array}$} \\
\hline Muy satisfecho & Satisfecho & Neutro & Insatisfecho & $\begin{array}{c}\text { Muy } \\
\text { insatisfecho }\end{array}$ \\
\hline $34,4 \%$ & $31,1 \%$ & $26,2 \%$ & $6,6 \%$ & $1,7 \%$ \\
\hline \multicolumn{5}{|c|}{ ¿Considera que el clima laboral de la institución se ha deteriorado durante la pandemia? } \\
\hline \multicolumn{3}{|c|}{$\mathrm{Si}$} & \multicolumn{2}{|c|}{ No } \\
\hline \multicolumn{2}{|c|}{$45,9 \%$} & & \multicolumn{2}{|c|}{$54,1 \%$} \\
\hline
\end{tabular}

La valoración positiva y negativa de la dimensión satisfacción es:

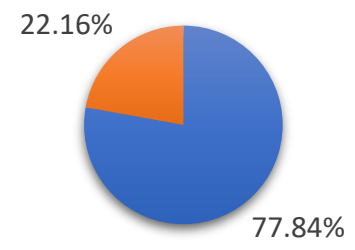

Positivo Negativo

Figura 4. Valoración de la dimensión satisfacción

Cabe mencionar que no se tomaron en cuenta los porcentajes de la pregunta 3 , ya que no es posible indicar alguna preferencia como positiva o negativa. 
En esta última dimensión interna la valoración es positiva, lo cual indica que existe una elevada satisfacción de los docentes en la realización de las actividades laborales.

\section{Dimensión neutra}

La eficacia es entendida como la consecución de metas, los logros de los objetivos propuestos, evidenciándose una capacidad administrativa para alcanzar los resultados, de tal manera que impactan en la productividad y en la efectividad de un equipo de trabajo. La eficiencia es el criterio económico que revela la capacidad administrativa de producir el máximo resultado con el mínimo de recurso, energía y tiempo, por lo que optimiza la utilización de los recursos disponibles para la obtención de resultados deseados (Rojas et al.,2018). Esta dimensión analiza desde el punto de vista de los colaboradores, los logros alcanzados vs los recursos empleados (incluyéndose la flexibilidad).

La eficacia y eficiencia fue determinada como la única dimensión neutra, indagándose en la percepción sobre la carga laboral, donde el 78,7\% estimó que es la correcta, por su parte el 39,3\% considera que las directrices son dadas de manera clara, el 49,2\% menciona que los plazos son los adecuados para la realización de las actividades laborales y el 70,5\% indica que existe flexibilidad para entregar asignaciones atrasadas (tabla VI).

Tabla VI. Resultado de la dimensión neutra eficacia y eficiencia

\begin{tabular}{|c|c|c|}
\hline \multicolumn{3}{|c|}{ Considera que la carga laboral docente es la idónea para usted? } \\
\hline \multirow{2}{*}{\multicolumn{2}{|c|}{ Sí }} & No \\
\hline & $78,7 \%$ & $21,3 \%$ \\
\hline \multicolumn{3}{|c|}{ ¿Las directrices para realizar un determinado trabajo asignado son claras? } \\
\hline Sí & A veces & No \\
\hline $39,3 \%$ & $55,8 \%$ & $4,9 \%$ \\
\hline \multicolumn{3}{|c|}{$\begin{array}{l}\text { ¿Los plazos dados para realizar y entregar un trabajo están proporcionalment } \\
\text { establecidos? }\end{array}$} \\
\hline Sí & A veces & No \\
\hline $49,2 \%$ & $41 \%$ & $9,8 \%$ \\
\hline \multicolumn{3}{|c|}{ ¿Existe flexibilidad para la entrega de trabajo atrasado que le fue asignado? } \\
\hline Sí & A veces & No \\
\hline $70,5 \%$ & $27,9 \%$ & $1,6 \%$ \\
\hline
\end{tabular}

La valoración positiva y negativa de la dimensión eficacia y eficiencia es:

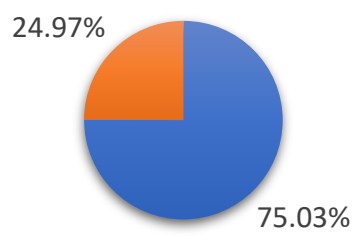

Positivo Negativo

Figura 5. Valoración de la dimensión eficacia y eficiencia 
La valoración en la dimensión eficacia y eficiencia indicaría que existe proporcionalidad entre las actividades, las directrices y los plazos, además de flexibilidad en la entrega de las asignaciones laborales.

\section{Dimensiones externas}

El liderazgo organizacional es el proceso mediante el cual una persona influye en otros para lograr los objetivos comunes a corto y largo plazo. Esta característica puede ser aprendida, sin embargo, las habilidades y conocimientos pueden ser influenciados por las creencias, los valores, la ética y el carácter (Cuartas, 2010). En base a lo anterior se indagó sobre la percepción de liderazgo proyectado en la institución objeto de estudio, donde el 39,3\% de las personas indica que son interrumpidos regularmente fuera de sus horas laborales (tabla VII), existiendo una clara violación a la "Ley de Apoyo Humanitario" que fue aprobada en al año 2020 en Ecuador, donde se menciona que "el empleador debe respetar este derecho (de la desconexión), garantizando el tiempo en el cual el colaborador no estará obligado a responder sus comunicaciones, órdenes u otros requerimientos" (El Comercio, 2020), esta desconexión deberá ser de al menos 12 horas continuas, en un período de 24 horas. Es importante señalar que el $63,9 \%$ de los encuestados considera que su jefe directo es justo en la toma de las decisiones, mientras $55,7 \%$ indica que existen sesgos de las autoridades para beneficiar a ciertas personas.

Tabla VII. Resultado de la dimensión externa liderazgo

\begin{tabular}{|c|c|c|}
\hline \multicolumn{3}{|c|}{ ¿De manera regular es interrumpido en sus horas fuera de la jornada laboral? } \\
\hline Sí & A veces & No \\
\hline $39,3 \%$ & $47,5 \%$ & $13,2 \%$ \\
\hline \multicolumn{3}{|c|}{ ¿Su jefe directo es justo en sus decisiones? } \\
\hline Sí & A veces & No \\
\hline $63,9 \%$ & $34,4 \%$ & $1,7 \%$ \\
\hline ¿Considera que existen sesgos de parte de las autoridades para favorecer a ciertas \\
personas?
\end{tabular}

La valoración positiva y negativa de la dimensión liderazgo es:

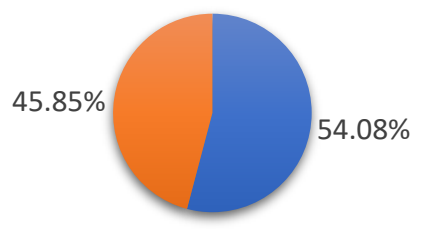

- Positivo Negativo

Figura 6. Valoración de la dimensión liderazgo 
Se consideró en esta dimensión la interrupción de las horas de descanso, dado que las decisiones son tomadas por los jefes inmediatos o por las autoridades institucionales.

La valoración del liderazgo es positiva, sin embargo, los factores negativos se encuentran próximos al margen de error del 5\%, es decir, matemáticamente son valores diferentes, pero estadísticamente son iguales. Por lo tanto, se debe fortalecer esta dimensión dentro de la institución.

El reconocimiento laboral es una dimensión fundamental para que los colaboradores se sientan parte de la institución, proporcionándole una satisfacción personal que les hace desarrollar su trabajo de forma positiva. Según Gitman y Mc. Daniel (2008) el reconocimiento laboral es una retroalimentación, donde se motiva y refuerza positivamente a los empleados, ya que estos necesitan conocer que están realizando un buen desempeño en sus funciones.

El $44,3 \%$ de los docentes considera que han recibido el justo reconocimiento de su esfuerzo, mientras que el $37,7 \%$ menciona que en ocasiones lo ha recibido (tabla VIII).

Tabla VIII. Resultado de la dimensión externa reconocimiento

\begin{tabular}{|c|c|c|}
\hline \multicolumn{2}{|c|}{ ¿Considera que su esfuerzo ha tenido el justo reconocimiento? } \\
\hline Sí & A veces & No \\
\hline $44,3 \%$ & $37,7 \%$ & $18 \%$ \\
\hline
\end{tabular}

La valoración positiva y negativa de la dimensión reconocimiento es:

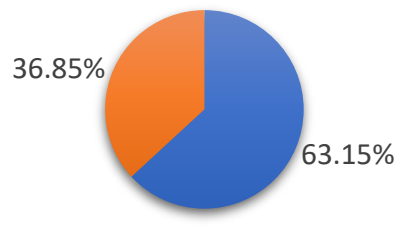

- Positivo Negativo

Figura 7. Valoración de la dimensión reconocimiento

La valoración de la dimensión reconocimiento es positiva, donde se infiere que las autoridades reconocen el esfuerzo de todos los miembros de la institución educativa.

Las oportunidades para el desarrollo profesional efectivo mejora la satisfacción de las personas, eleva su autoestima, permite reducir el estrés y fortalece la salud psicológica y física de los colaboradores o empleados. Este desarrollo profesional dentro de la institución beneficia a ambas partes, ya que los empleados se adaptan de manera más rápida y mejor a las necesidades, y la organización mejora los niveles de productividad y se crean las condiciones para la innovación (Inesem, 2018). 
El 78,7\% de los encuestados considera que existen las condiciones necesarias para crecer profesionalmente en la institución, mientras que el $63,9 \%$ estima que pueden realizar innovaciones dentro de sus funciones laborales (tabla IX).

Tabla IX. Resultado de la dimensión externa innovación

\begin{tabular}{|c|c|c|}
\hline \multicolumn{3}{|c|}{ ¿Cree que tiene oportunidades para crecer profesionalmente en la institución? } \\
\hline Sí & & \\
\hline $78,7 \%$ & & \\
\hline \multicolumn{3}{|c|}{ ¿Ha tenido oportunidades de innovar en su trabajo? } \\
\hline Sí & A veces & No \\
\hline $63,9 \%$ & $29,5 \%$ & $6,6 \%$ \\
\hline
\end{tabular}

La valoración positiva y negativa de la dimensión oportunidades de desarrollo e innovación es:

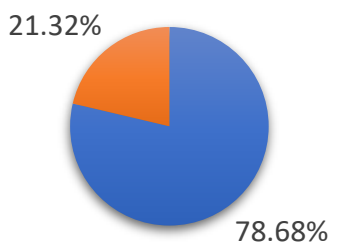

Positivo Negativo

Figura 8. Promedio de las valoraciones positivas y negativas de las siete dimensiones

La dimensión oportunidades de desarrollo e innovación tiene una puntuación general determinada como alta, con ello se deduce que existen las condiciones internas para que los colaboradores puedan crecer profesionalmente y crear mecanismos que terminen en innovaciones.

Con los porcentajes anteriormente indicados, se diseña el siguiente gráfico donde se detallan las valoraciones positivas y negativas de cada una de las dimensiones: 


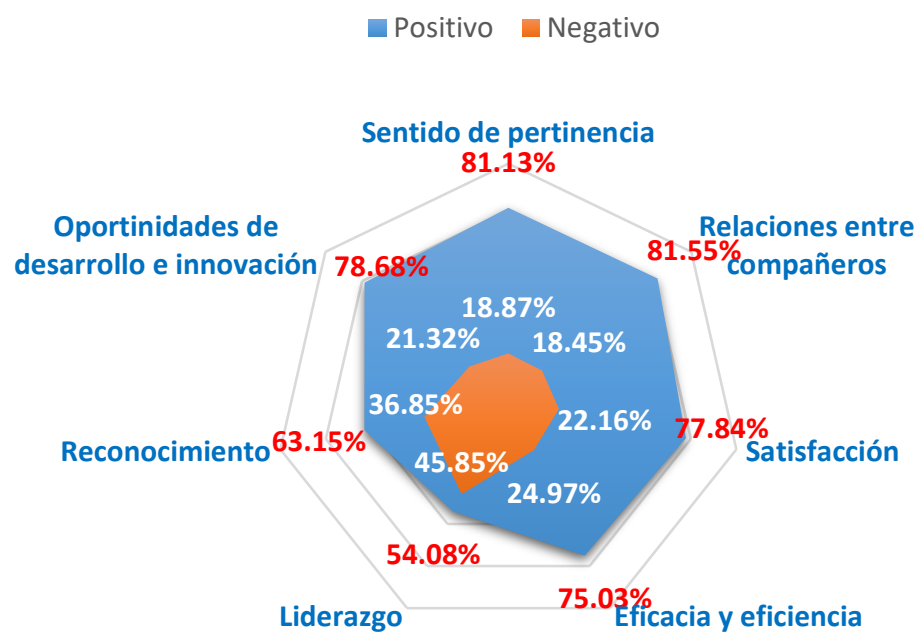

Figura 9. Resumen de las valoraciones de cada una de las dimensiones

La valoración final entre las siete dimensiones es la siguiente:

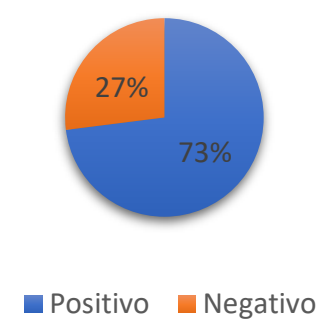

Figura 10. Valoración de las 7 dimensiones

Finalmente, se les preguntó a los docentes participantes sobre su percepción del actual clima laboral institucional. El 54,1\% lo estimó como satisfactorio, el 24,6\% como muy satisfactorio y el 21,3\% como regular (figura $11)$.

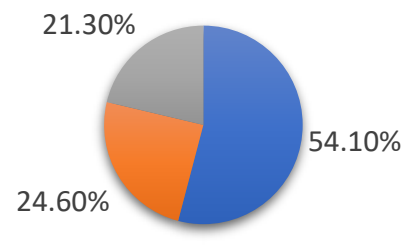

- Muy satisfactorio $\quad$ Satisfactorio $\quad$ Regular

Figura 11. Clima laboral de manera general según los docentes participantes 


\section{Entrevista a la autoridad máxima de la institución}

La doctora Ida Campi Mayorga, rectora de la institución concedió una entrevista el jueves 25 de marzo, donde se resaltan los siguientes comentarios en los diferentes ejes abordados:

- Valores de un líder: el compromiso y la responsabilidad son los principales valores que debe tener un líder institucional, donde no se descartan otros tipos de valores, no obstante, sobre estos dos giran los demás, los cuales consienten buenos resultados de manera colectiva.

- Sostenimiento del buen liderazgo en medio de la pandemia: la transformación de los hábitos laborales repercutió de manera significativa en la institución, donde el equipo docente pudo responder de manera positiva a las nuevas exigencias, sin embargo, en la parte operativa debido a las restricciones de movilidad, estas afectaron las operaciones normales de la institución (no significativamente). Todo esto demuestra que no existe una receta única para mantener un liderazgo, sino que él mismo es la suma de todos los esfuerzos internos.

- Calidad laboral en teletrabajo: no se considera que la realización de las actividades laborales dentro del hogar haya afectado en la calidad. Las actividades propias del hogar (principalmente en las mujeres) son un factor que pueden generar estrés en las personas, pudiendo sentirse agobiados por la combinación de las actividades del hogar y laborales, pero con la particularidad que ambas convergen en el mismo espacio, ya que ahora no existe la barrera física que delimita ambas acciones.

- Las actividades virtuales y calidad laboral: en las actuales circunstancias no podría identificarse que la calidad laboral es peor o mejor, sino que podríamos hablar del grado de adaptación laboral, ya que no existen parámetros previos que permitan evaluar las actividades presenciales $v s$ las virtuales.

- Mejoramiento de las condiciones laborales: al realizarse las jornadas laborales de manera virtual, los docentes son los encargados de mejorar su entorno (hogar), de tal manera que les permita cumplir con sus actividades programadas. La institución responsable de su capital humano ha realizado varias capacitaciones virtuales en diferentes temáticas, con el objetivo de mejorar dichos entornos dentro de los hogares.

- Clima laboral en la pandemia: los elementos positivos no han disminuido, eso se evidencia a través de los diferentes resultados que la institución sigue alcanzando, a pesar de las circunstancias adversas presentadas. 


\section{Discusión}

Las dimensiones que componen el clima organizacional han sido caracterizadas por muchos investigadores, no existiendo un consenso para establecerlas de manera general, ya que muchos consideran las particularidades únicas dentro de un ambiente social. Por ejemplo, Cabrera (1999) considera que los elementos que configuran el clima laboral dependen de la percepción de los miembros de esta, entre las que se encuentran las variables del ambiente físico, estructurales y las propias del comportamiento de la organización. Patterson et al. (2005), establece las siguientes dimensiones: bienestar de los empleados, autonomía, participación, comunicación, énfasis en el entrenamiento, integración, apoyo de la supervisión, formalización, tradición, flexibilidad, innovación, foco en lo exterior, reflexión, claridad, esfuerzo, eficiencia, calidad, presión, retroalimentación. Taylor y Bowers (1972), estudiaron las siguientes dimensiones laborales: apertura a los cambios tecnológicos, recursos humanos, comunicación, motivación y la toma de decisiones. Litwin y Stringer (1968), definieron a través de un cuestionario nueve dimensiones que se relacionan con las propiedades de la organización y que explicarían las principales interacciones dentro de la misma, las cuales son: estructura, responsabilidad, recompensa, desafío, relaciones, cooperación, estándares, conflictos e identidad.

En base a lo anterior, se puede inferir que la dimensionalidad en el análisis del clima laboral es algo subjetivo para cada investigador y que los elementos establecidos para medirlo varían según las particularidades existentes dentro de un lugar o ambiente. Por lo tanto, no existe un estándar de medición, sino un conjugado de elementos que se deben considerar, los cuales dependen de los factores que se desean establecer.

Para Moreira-Moreira (2016), "la importancia del clima organizacional en la formación de recursos humanos en la educación superior; se reflexiona sobre diferentes formas de determinación de este clima, el liderazgo, en donde se explora la experiencia ecuatoriana para el cambio organizacional de las instituciones de educación superior como pilar fundamental para el mejoramiento de su calidad académica". También menciona que el análisis de los estudios del clima organizacional en la gestión del recurso docente en la educación superior permite generar información oportuna y necesaria para lograr identificar las necesidades en relación con el futuro deseado dentro de la institución, y con ello trazar las estrategias y acciones pertinentes.

En base a los enunciados anteriores, se reafirma la importancia del clima laboral en una institución de educación superior, donde los hallazgos de la presente investigación ayudan a deducir que este elemento sigue teniendo aspectos altos que ayudan a desarrollar las diferentes actividades 
institucionales. Claramente la investigación realizada analizó la percepción de los docentes desde la virtualidad de las actividades, tampoco existen parámetros anteriores para comparar los resultantes entre la presencialidad y con esa nueva forma laboral, sin embargo, se infiere que la interrelación entre las personas y la institución se encuentra fortalecida a pesar de los distanciamientos físicos causados por la pandemia.

\section{Conclusiones}

En base a los fundamentos de las revisiones bibliográficas, se establecieron 7 dimensiones para determinar el clima laboral institucional, las cuales son: sentido de pertenencia, relaciones entre compañeros, satisfacción, eficacia y eficiencia, liderazgo, reconocimiento y oportunidades de desarrollo e innovación. Estas fueron subdivididas en internas, neutra y externas. Estas dimensiones tratan de abarcar las principales interrelaciones que desarrollan los docentes dentro de la institución objeto de estudio.

Luego de la aplicación de las encuestas realizadas a los docentes del Instituto Superior Tecnológico Juan Bautista Aguirre, se logró determinar que el clima laboral de la institución es satisfactorio, ya que, más de la mitad de los encuestados concordaron con esta postura. La estimación porcentual de las 7 dimensiones permitió establecer que existe una valoración positiva equivalente al $73 \%$ de las opiniones y un $27 \%$ de las percepciones fueron negativas. El primer valor representa la determinación del clima laboral a través de la estimación favorable de los porcentajes en cada una de las dimensiones analizadas. La valoración positiva más alta proviene de la dimensión relación entre compañeros, mientras que la valoración negativa más alta pertenece a la dimensión liderazgo. Al aplicarse el criterio de positividad para las opiniones de muy satisfactorio y satisfactorio en los resultados de la pregunta 21 , se denota que el $78,7 \%$ de los participantes considera como altamente favorable el actual clima laboral durante las jornadas de teletrabajo.

La entrevista con la rectora de la institución permitió conocer la percepción de la gerencia en la relación a los valores del liderazgo, la calidad laboral durante la pandemia, aportes de la institución para mejorar las condiciones laborales dentro de los hogares y en la estimación cualitativa del actual clima laboral institucional, siendo este último elemento favorable desde la visión de la entrevistada. Esto permitió contrastar la opinión de los docentes y de las autoridades de la institución objeto de estudio, donde ambos grupos estiman como positivo el clima laboral, lo que fomenta el correcto desempeño de las labores institucionales.

La investigación permitió conocer la percepción de los docentes en un "clima laboral virtual", el mismo que lógicamente posee muchos contrastes con el presencial. Sin embargo, existen la noción generalizada que es posible mantener un ecosistema laboral positivo que permita realizar las actividades 
regulares a pesar de la barrera que implica la no presencialidad. Los resultados no son comparativos con otros estudios, sino que intentan dejar la semilla teórica para futuros análisis comparativos.

\section{References:}

1. Acosta, B., \& Venegas, C. (2010). Clima organizacional en una empresa cervecera: un estudio exploratorio. Revista de Investigación en Psicología -, 13(1), 163-172.

2. Bizneo. (2021). 7 indicadores del clima laboral. Obtenido de https://www.bizneo.com/blog/7-indicadores-del-clima-laboral/

3. Bobadilla, C. (2017). Clima organizacional y desempeño laboral en institutos superiores tecnológicos de Huancayo. Perú: Universidad Nacional del Centro de Perú.

4. Cabrera, G. (1999). Clima organizacional dos empresas chilenas. Revista de Psicología Social e Institucional, 1(2), 1-19.

5. Chiavenato, I. (2009). Gestión del Talento Humano. Mc. Graw Hill.

6. Condella, C. (2012). ¿Por qué no podemos ser amigos virtuales? Comunicación(138).

Obtenido

de https://reunir.unir.net/bitstream/handle/123456789/5513/Por\%20que $\% 20$ no $\% 20$ podemos $\% 20$ ser $\% 20$ amigos $\% 20$ virtuales.pdf?sequence $=$ $1 \&$ isAllowed $=\mathrm{y}$

7. Cuartas, C. (2010). Factores que afectan el clima laboral de la imprenta departamental de Antioquía, durante el año 2010. Colombia: Universidad Minuto de Dios.

8. Daft, R. (2011). Teoría y diseño organizacional. Cengage Learning Editores,.

9. Dessler, G., \& Varela, R. (2011). Administración de recursos humanos. Pearson.

10. El Comercio. (2020). ¿En qué consiste el derecho a 12 horas de desconexión que tiene el teletrabajador en Ecuador? Obtenido de https://www.elcomercio.com/actualidad/derecho-desconexionteletrabajo-ecuador-explicacion.html

11. García, I. (2006). La formación del clima psicológico y su relación con los estilos de liderazgo. Granada, España: Universidad de Granada.

12. Gitman, L., \& Mc. Daniel, C. (2008). El futuro de los negocios. Cengage Learning.

13. Inesem. (2018). Plan de desarrollo profesional y su importancia para las empresas. Obtenido de https://revistadigital.inesem.es/gestionempresarial/plan-de-desarrollo-profesional-y-su-importancia-paralas-empresas/

14. Jurado, A. (2014). Los niveles de sentido de pertenencia en un grupo de profesionales bajo contratación laboral simulada en distintas 
organizaciones guatemaltecas. Guatemala: Universidad Rafael Landívar.

15. Litwin, G., \& Stringer, R. (1968). Motivation and Organizational Climate. Boston, MA: Harvard Business School Press.

16. Méndez, M., Olvera, M., Cabrera, A., Ordoñez, J., Buendía, D., \& Guilcapi, M. (2020). Evaluación de la Virtualización del Aprendizaje y los Desafíos en la Educación Superior en el Instituto Superior Tecnológico Juan Bautista Aguirre. European Scientific Journal, 16(31), 81-111. doi:10.19044/esj.2020.v16n31p81

17. Miguel, J. (2020). La educación superior en tiempos de pandemia: una visión desde dentro del proceso formativo. Revista Latinoamericana de Estudios Educativos, L, 13-40. Obtenido de redalyc.org/jatsRepo/270/27063237017/html/index.html

18. Moreira-Moreira, L. (2016). Clima Organizacional en la Educación Superior, Ecuador. Dominio de las Ciencias, 2(4), 296-307.

19. Moreno, S., \& Pérez, E. (2018). Relaciones interpersonales en el clima laboral de la universidad tecnológica del Chocó Diego Luis Córdoba. Revista CES Derecho, 9(1), 13-33.

20. Patterson, M., West, M., Shackleton, V., Dawson, J., Lawthom, R., Maitlis, S., . . . Wallace, A. (2005). Validating the organizational climate measure: links to managerial practices, productivity and innovation. Journal of Organizacional Behavior, 26, 379-408.

21. Revista Líderes. (2018). El clima laboral es la inquietud de siempre. Obtenido de https://www.revistalideres.ec/lideres/clima-laboralinquietud-siempre.html

22. Taylor, J., \& Bowers, D. (1972). Survey of organizations: A machinescored standardized questionnaire instrument. Center for Research on Utilization of Scientific Knowledge, University of Michigan. 